\title{
Molecular Survey and Phylogenetic Analysis of Atypical Porcine Pestivirus (APPV) Identified in Swine and Wild Boar from Northern Italy
}

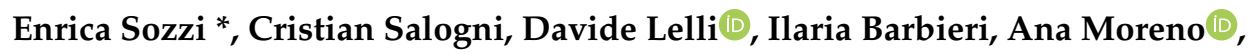 \\ Giovanni Loris Alborali and Antonio Lavazza \\ Istituto Zooprofilattico Sperimentale della Lombardia e dell’Emilia Romagna “Bruno Ubertini” (IZSLER), \\ Via Antonio Bianchi 7/9, 25124 Brescia, Italy; cristian.salogni@izsler.it (C.S.); davide.lelli@izsler.it (D.L.); \\ ilaria.barbieri@izsler.it (I.B.); anamaria.morenomartin@izsler.it (A.M.); giovanni.alborali@izsler.it (G.L.A.); \\ antonio.lavazza@izsler.it (A.L.) \\ * Correspondence: enrica.sozzi@izsler.it; Tel.: +39-030-2290361
}

Received: 14 November 2019; Accepted: 8 December 2019; Published: 10 December 2019

\begin{abstract}
Atypical porcine pestivirus (APPV) is a newly recognized member of the Flaviviridae family. This novel porcine pestivirus was first described in 2015 in the USA, where it has been associated with congenital tremor type A-II in new-born piglets. APPV is widely distributed in domestic pigs in Europe and Asia. In this study, a virological survey was performed in Northern Italy to investigate the presence of APPV using molecular methods. Testing of 360 abortion samples from pig herds revealed two APPV strains from distinct provinces in the Lombardy region and testing of 430 wild boar blood samples revealed three strains, one from Lombardy and two from Emilia Romagna. The nucleotide sequencing of a fragment of the nonstructural protein 3-coding region revealed a high similarity to the previously detected European strains (Spanish, German, and Italian) of APPV.
\end{abstract}

Keywords: pestivirus; pig; APPV; phylogenetic analysis; Italy

\section{Introduction}

Pestiviruses are highly variable single-stranded RNA genome viruses, belonging to the Flaviviridae family. Actually, based on molecular and epidemiological evidence, the genus Pestivirus includes eleven species, indicated with progressive letters from A to K. Thus, the "classical" species are A (Bovine viral diarrhea virus 1), B (Bovine viral diarrhea virus 2), C (Classical swine fever virus), and D (Border disease virus), whereas the new species are $\mathrm{E}$ to $\mathrm{K}$ [1]. In addition, other pestiviruses were described, likely as three additional species, respectively, in bats (bat-derived pestivirus) [2,3], sheep and goats (Tunisian sheep pestiviruses) [4], and pigs (Linda pestivirus) [5]. In the swine species only, in addition to Pestivirus C, three pestiviruses have been identified to date: (1) Pestivirus F (Bungowannah virus) reported only in Australia, as a cause of reproductive disorders, fetal death, and sudden death in piglets, [6]; (2) The Linda virus described in association with congenital tremors (CTs) in piglets in Austria and thereafter only occasionally reported, leaving its geographical spread and clinical relevance in pigs undefined [5]; and (3) Pestivirus K, commonly known as atypical porcine pestivirus (APPV), which is the most relevant due to the frequency of identification, clinical findings, and economic importance. In fact, it has been identified several times in North America [7,8], South America [9,10], Europe [11-13], and Asia [14,15], and it should be considered as stably present for a long time in domestic and wild pig populations $[8,10,15,16]$. This is true also for Italy, since in a previous survey, at least four APPV isolates were found between 2015-2017 and a quite high seroprevalence was detected in pig sera [15]. 
Although APPV has been repeatedly identified in asymptomatic animals, there is clear evidence that it is associated with CT syndrome type A-II (CT A-II) in newborns [7]. Clinically healthy pigs and wild boars may have an epidemiological role as vehicles of APPV, but, considering the different frequency of detection in wild boars, which was quite high in Germany and Serbia [17] and very low in Spain [18], the epidemiology of APPV may vary considerably from country to country with increases in livestock and wild populations, animal breeding, and world trade [18]. While one study described the economic losses caused by a 10\% drop in the number of weaned piglets per sow [19], the full economic consequences of APPV outbreaks remain to be determined.

In this study, the presence of the APPV genome in pig fetuses and wild boars from both the Lombardy and Emilia Romagna regions of Northern Italy was determined, and the genetic characterizations of the identified strains are described.

\section{Materials and Methods}

\subsection{Pigs}

From 2016 to 2018, 360 fetuses of pigs from pig farms in the Lombardy region were examined at the IZSLER Diagnostic Laboratory in Brescia. All of the samples examined originated from field cases of spontaneous abortions in pig farms and sent to the general diagnostic laboratory of IZSLER to determine the presence of any infectious agent. In none of these cases was the presence of clinical signs referable to CT syndrome specifically reported. During necropsy, samples of organs (brain, lung, spleen, liver, and kidney) were taken from each aborted fetus, then collected into a single farm-specific pool and homogenized (10\% w/v) in minimum essential medium (MEM; Gibco, Life Technologies, Paisley, UK) supplemented with an antibiotic (1000 U/mL penicillin, $1 \mathrm{mg} / \mathrm{mL}$ streptomycin; Gibco, Life Technologies, Paisley, UK) and anti-mycotic $(2.5 \mu \mathrm{g} / \mathrm{mL}$ amphotericin B; Gibco, Life Technologies, Paisley, UK). After centrifugation, the supernatant was analyzed to identify any agents that cause abortions in swine. For bacteriological agents such as Brucella spp., Listeria spp., and Mycoplasma spp., the screening and pathogen identification were conducted according to Office International des Epizooties (OIE) standardized protocols [20]. The presence of Chlamydophila spp. was investigated by real-time Polymerase Chain Reaction (PCR) directly in biological samples [21] and typing by the PCR-restriction fragment length polymorphism (RFLP) assay, targeting the $16 \mathrm{~S}$ ribosomal gene [22]. For Mycoplasma spp., the PCR method described by van Kuppeveld et al. [23] was used. Virological analyses for detecting the more common pig pathogens were conducted using a panel of PCR methods including porcine reproductive and respiratory syndrome virus (PRRSV) (AgPath-ID ${ }^{\mathrm{TM}} \mathrm{NA}$ and EU PRRSV Multiplex@ Applied Biosystems), porcine circovirus type 2 (PCV-2) [24], porcine parvovirus (PPV) [25], and porcine circovirus type 3 (PCV-3) [26]. The presence of pestiviruses was determined by using a pan-pestivirus real-time RT-PCR [27], and, considering its limited capacity in detecting APPV, by a APPV-specific real-time RT-PCR [19]. In addition, all the tested samples were inoculated on cell cultures (primary embryonic swine kidney cells, swine alveolar macrophages, and monkey kidney cell line MARC-145), which allow the isolation of a broad range of swine viruses. The inoculated cell monolayers were observed daily for 5-7 d for the appearance of a cytopathic effect and then sub-cultured to the second passage, at which time they were independently tested using an "in-house" sandwich enzyme-linked immunosorbent assay (ELISAs) for the presence of pestivirus [28] and PRRSV antigens [29]. Only those samples that were positive for APPV at the initial screening test using the APPV-specific real-time RT-PCR were further subcultured to improve the chance to isolate the APPV until the fifth passage, at which time, even in the absence of a cytopathic effect, they were assessed again with the APPV-specific real-time RT-PCR.

\subsection{Wild Boars}

In total, 430 blood samples of wild boars, killed during the 2017-2018 hunting season, were collected in the framework of the Lombardy and Emilia Romagna wildlife monitoring plans for 
classical swine fever (CSF) and Aujeszky disease and transferred to IZSLER for examination. Serum samples were tested for antibodies against swine vesicular disease virus [30,31], encephalomyocarditis virus [32], glycoprotein E of Aujeszky's disease virus [33], pestivirus (A-D) [34], swine influenza virus type A, subtypes H1N1, H1N2, and H3N2 [35,36], and finally for Brucella spp. (Svanovir Brucella-Ab C-ELISA(C). Serological analyses were conducted with the methods currently in use at the IZSLER.

\subsection{Identification and Genomic Characterization of Atypical Porcine Pestivirus (APPV)}

For the investigation of the APPV genome, all samples, both the homogenates from fetal organs and the wild boar sera, were screened using the NS5B gene-specific real-time RT-PCR method [19]. Samples that tested positive were characterized by Sanger sequencing using RT-PCR that amplified a fragment of the NS3 region. The nucleotide sequences were aligned using the ClustalW method and compared with sequences present in GenBank [37] using MEGA6 software [38]. The maximum likelihood phylogenetic tree was constructed using IQ-tree software [39] by applying the TIM2+F+G4 model identified using ModelFinder selection [40].

\section{Results}

\subsection{Pigs}

The examination of the NS5B gene by real-time RT-PCR in the homogenized samples of pig fetuses identified two $(0.6 \%)$ positive samples in two distinct farrow-to-finish farms, one from the province of Brescia in a pool containing the organs of three fetuses and the other from the province of Mantua in a pool containing the organs of two fetuses (Figure 1).

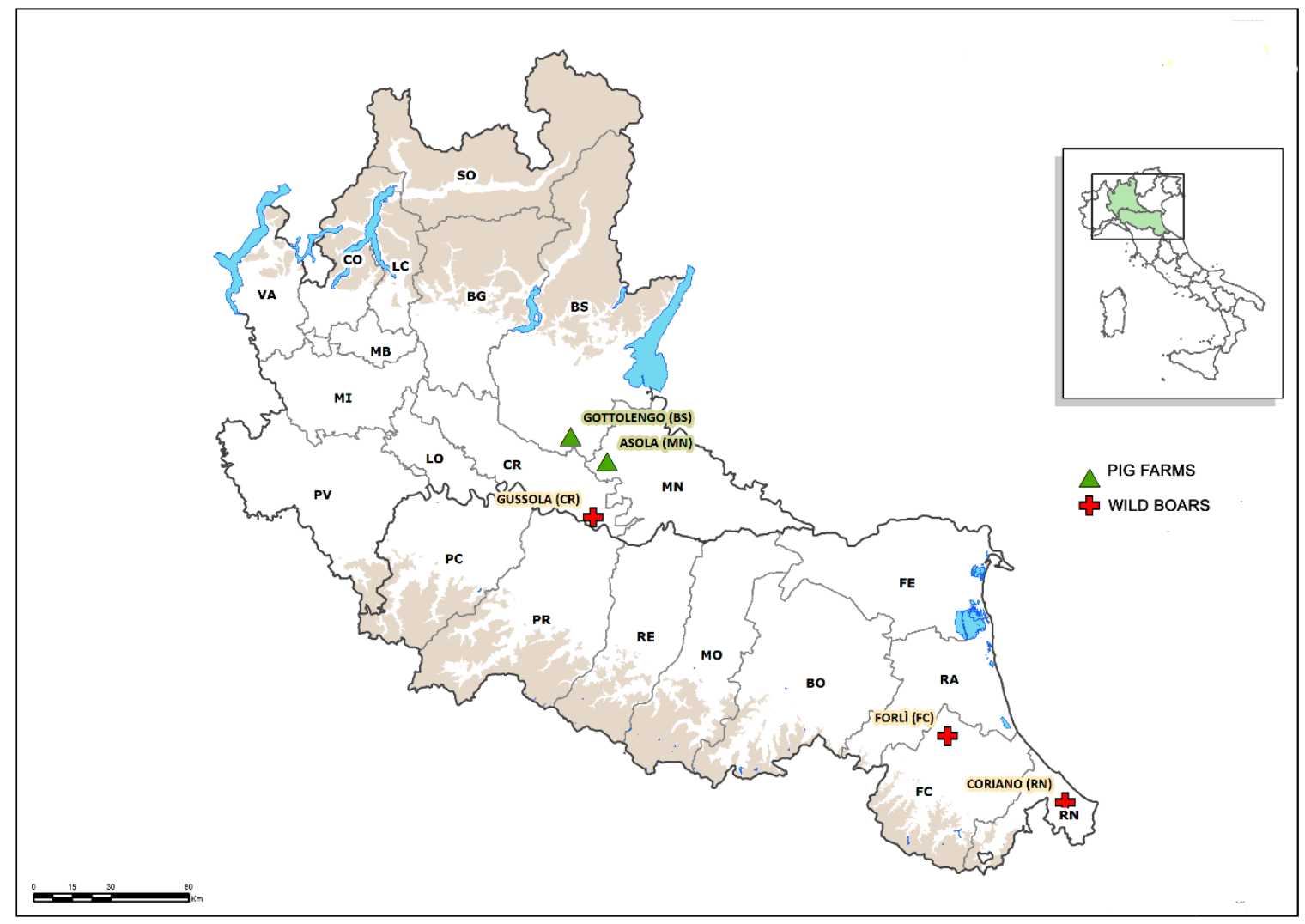

Figure 1. Geographical distribution of the pig farms and wild boar hunting sites where atypical porcine pestivirus (APPV) was identified.

At necropsy, none of these fetuses showed internal macroscopic lesions, and bacteriological investigations consistently produced negative results. Moreover, the molecular examinations for 
PRRSV, PCV-2, PPV, and pan-pestivirus were all negative, and only the real-time PCR for PCV-3 produced a positive result from a pool of fetuses that originated from the province of Brescia. The two sequences obtained, APPV_Italy_SW_BS341729_2017 and APPV_Italy_SW_MN212160_2016, had a nucleotide similarity of $92.5 \%$ between them and clustered with APPVs previously identified in Europe. In particular, the phylogenetic tree constructed using the NS3 region (Figure 2) revealed that the two identified strains belong to distinct groups: (1) the APPV_Italy_SW_MN212160_2016 strain is related to both the 98/Sp06 strain identified in Spain in 2006 [16] (99.2\% identity) and the German strain Bavaria S5/9 identified in 2015 [11] (96.8\% identity); and (2) APPV_Italy_SW_BS341729_2017 forms a separate and closely related clade with two sequences identified in 2015 in pigs from Italy, Italy-164 and Italy-181 [15], with a nucleotide similarity of $95.5 \%$ and $99.2 \%$, respectively.

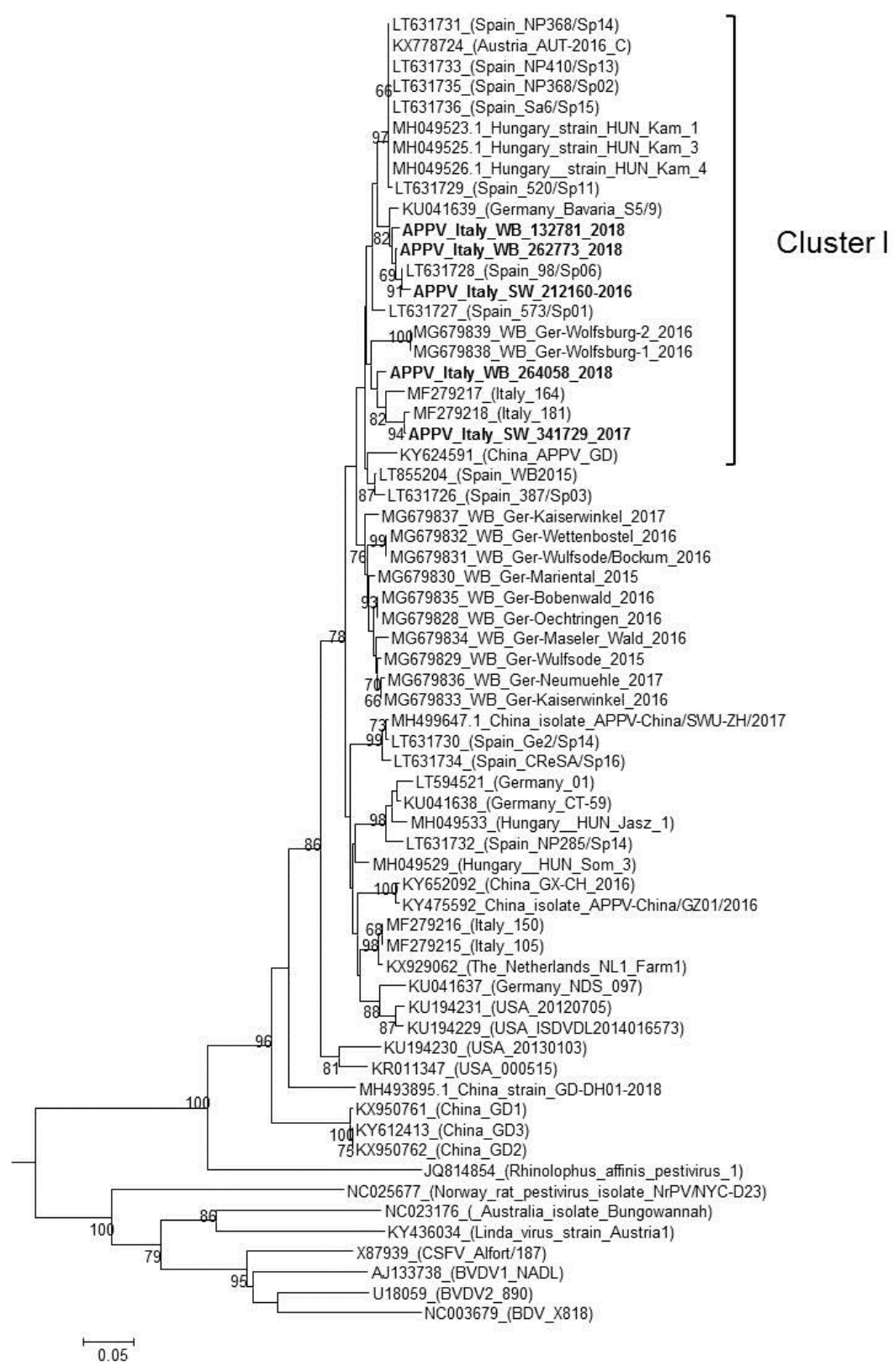

Figure 2. Phylogenetic tree based on a 645-nt fragment of the nonstructural protein 3-encoding region of the atypical porcine pestivirus (APPV) genome present in GenBank. A phylogenetic analysis using the maximum likelihood method including 1.000 bootstrap iterations was performed. Only bootstrap values $\geq 60$ are indicated. Sequences in bold were generated in this study. 
Despite the positive results of the real-time RT-PCR test, virological examinations of the organs of swine fetuses by inoculating cell cultures up to the fifth passage produced consistent negative results. Consequently, we did not succeed in isolating any APPV strains.

\subsection{Wild Boars}

All of the wild boar samples examined in this study including those that tested APPV-positive, originated from hunting activity, and thus, they were considered healthy based on their behavior before shooting and the absence of lesions on carcasses examined at slaughter. Out of the 430 blood samples examined, three $(0.69 \%)$, originating from the Cremona Province in Lombardy (APPV_Italy_WB_CR264058_2018) and from two neighboring provinces, Rimini and Forlì in Emilia Romagna (APPV_Italy_WB_RN262773_2018 and APPV_Italy_WB_FC132781_2018, respectively) (Figure 1), screened positive for APPV. In agreement with the genomic characterization of the strains identified in pigs, the sequences of those identified in wild boars could be divided into two distinct clusters. The first included the two strains detected in Emilia Romagna (APPV_Italy_WB _RN262773_2018 and APPV_Italy_WB_FC132781_2018) and the sequence identified in the pig farm from Mantua Province in Lombardy (APPV_Italy_SW_MN212160_2016), which had identities of 95.6\% and $96 \%$, respectively; plus the previously characterized strains BavariaS5/9 and Spain 98/Sp06, with which the WB strains had a higher nucleotide identity of 98-99.2\%.

The second cluster included the APPV_Italy_WB_CR264058_2018, the APPV Italy_SW_BS341729_2017 (96.4\% identity), the Italian pig strains, Italy-164 and Italy-181, which had nucleotide of $96.4 \%$ and $96 \%$, respectively, and the strains identified in 2016 in Lower Saxony, Germany (94.3\% identity).

For the collateral serological examinations, all APPV positive samples were negative for all serological tests employed except for: (a) one serum (APPV_Italy_WB_CR264058_2018) that tested positive for anti-Brucella spp. antibodies and (b) one (APPV_Italy_WB_FC132781_2018) that tested positive for glycoprotein E antibodies to the Aujeszky's disease virus.

The nucleotide sequences of the five APPV isolates were deposited in GenBank (NCBI) with the accession numbers MN736974-MN736978.

\section{Discussion}

The examination of 360 swine fetuses and 430 hunted wild boar blood samples identified APPVs in the area, two strains from the former and three strains from the latter. Based on the phylogenetic analysis performed, all five of these strains clustered with APPV strains previously identified in Spain and Germany. Although all of the sequences obtained can be grouped into the putative Cluster I described by Muñoz-González et al. [16], which should include viruses that have a common origin, the strains identified here could be further divided in two distinct sub-clusters.

Based on the absence of lesions on both fetuses and wild boars, we were keen to exclude a definite pathogenic role for APPV in these specific cases. However, it remains to be clarified whether the PCV-3 plus APPV (APPV_Italy_SW_BS341729_2017) co-infection, which was detected in the pool of fetuses originating from Brescia Province, may have had a synergistic effect in infected piglets, resulting in a clinical form characterized by abortion and natimortality. The very low prevalence of APPV found in the examined pig samples $(0.6 \%)$ is largely different from the sole previous study on APPV in Italy [15], which reported a higher prevalence of viral detection (17.5\%). This could be likely due to the sample types and selection, since we included in the survey only aborted pig fetuses with the aim of trying to establish if any correlation existed between the clinical case and the detection of APPV. A more specific monitoring program focused on the two APPV positive farms, and in general, to have systematically reported and analyzed cases of CT are anticipated in order to better define the prevalence of APPV in pig farms in Italy and clarify its effects.

Wild boars are susceptible to APPV infection, but their role in the epidemiology of the virus remains unknown. The low prevalence of APPV in the examined wild boar population is well correlated with the numbers reported by Colom-Cadena et al. [18] in Spain, but is in contrast to the 
high prevalence found in wild boars from northern Germany [17], where APPV seems to be endemic among wild boars in many areas. Considering the small number of strains isolated from the large geographical territory represented by the two regions (Lombardy and Emilia Romagna) and the lack of any particular geographic distribution of the clustered strains, it is impossible to draw epidemiological interpretations on APPV diffusion or on transmission between domestic pigs and wild boars.

\section{Conclusions}

APPV appears to be well established in the domestic swine populations of different countries in Europe, America, and Asia. Indeed, although identifying APPV has only become possible during the last few years due to the progressive refinement of diagnostic techniques, retrospective studies have indicated its circulation for many years. Based on the available genomic characterization data in the literature and the prevalence in domestic pigs, APPV exhibits a high genetic diversity among viral strains detected in different countries and tends to form independent clusters according to geographic locations. This study confirms the presence and distribution of APPV in populations of domestic and wild pigs present in the Lombardy and Emilia Romagna regions of Italy, which are under the jurisdiction of IZSLER. The high homology levels with strains identified in Germany and Spain reinforce the hypothesis that Italian strains have a European origin, and they confirm the likely determining role in the spread of infection being the commercial trade in pigs among different countries. The detection of new pestiviruses indicates the need to monitor for their presence and distribution using a systematic surveillance and diagnostic approach. In fact, the accurate and constant characterization of circulating strains is necessary to update the serological and virological tests, which in turn may be used to collect more detailed epidemiological information regarding APPV such as routes of entry and dissemination, and genetic evolution.

Author Contributions: C.S. and G.L.A. performed necropsy and routine laboratory examinations; E.S., I.B., D.L., and A.M. performed the laboratory work and analyzed the data; E.S., A.M., D.L., and A.L. interpreted the results and designed the figures; E.S. and A.L. wrote the manuscript. All the authors critically analyzed, revised, and approved the manuscript.

Funding: This study was supported by grants from the project PRC 2015019 of the Italian Ministry of Health.

Acknowledgments: The authors would like to thank Loredana Zingarello and Debora Campagna for their excellent technical assistance.

Conflicts of Interest: The authors declare no conflicts of interest.

\section{References}

1. Smith, D.B.; Meyers, G.; Bukh, J.; Gould, E.A.; Monath, T.; Scott Muerhoff, A.; Pletnev, A.; Rico-Hesse, R.; Stapleton, J.T.; Simmonds, P.; et al. Proposed revision to the taxonomy of the genus Pestivirus, family Flaviviridae. J. Gen. Virol. 2017, 98, 2106-2112. [CrossRef] [PubMed]

2. Wu, Z.Q.; Ren, X.W.; Yang, L.; Hu, Y.F.; Yang, J.; He, G.M.; Zhang, J.P.; Dong, J.; Sun, L.L.; Du, J.; et al. Virome analysis for identification of novel mammalian viruses in bat species from Chinese provinces. J. Virol. 2012, 86, 10999-11012. [CrossRef] [PubMed]

3. Wu, Z.Q.; Liu, B.; Du, J.; Zhang, J.P.; Lu, L.; Zhu, G.J.; Han, Y.L.; Su, H.X.; Yang, L.; Zhang, S.Y.; et al. Discovery of diverse rodent and bat pestiviruses with distinct genomic and phylogenetic characteristics in several Chinese provinces. Front. Microbiol. 2018, 9, 2562. [CrossRef] [PubMed]

4. Liu, L.L.; Xia, H.; Wahlberg, N.; Belák, S.; Baule, C. Phylogeny, classification and evolutionary insights into pestiviruses. Virol. 2009, 385, 351-357. [CrossRef] [PubMed]

5. Lamp, B.; Schwarz, L.; Högler, S.; Riedel, C.; Sinn, L.; Rebel-Bauder, B.; Weissenböck, H.; Ladinig, A.; Rümenapf, T. Novel pestivirus species in pigs, Austria, 2015. Emerg. Infect. Dis. 2017, 23, 1176-1179. [CrossRef]

6. Kirkland, P.D.; Frost, M.J.; Finlaison, D.S.; King, K.R.; Ridpath, J.F.; Gu, X. Identification of a novel virus in pigs-Bungowannah virus: A possible new species of pestivirus. Virus Res. 2007, 129, 26-34. [CrossRef] 
7. Arruda, B.L.; Arruda, P.H.; Magstadt, D.R.; Schwartz, K.J.; Dohlman, T.; Schleining, J.A.; Patterson, A.R.; Visek, C.A.; Victoria, J.G. Identification of a Divergent Lineage Porcine Pestivirus in Nursing Piglets with Congenital Tremors and Reproduction of Disease following Experimental Inoculation. PLoS ONE 2016, 11, e0150104. [CrossRef]

8. Hause, B.M.; Collin, E.A.; Peddireddi, L.; Yuan, F.; Chen, Z.; Hesse, R.A.; Gauger, P.C.; Clement, T.; Fang, Y.; Anderson, G. Discovery of a novel putative atypical porcine pestivirus in pigs in the USA. J. Gen. Virol. 2015, 96, 2994-2998. [CrossRef]

9. Gatto, I.R.H.; Harmon, K.; Bradner, L.; Silva, P.; Linhares, D.C.L.; Arruda, P.H.; de Oliveira, L.G.; Arruda, B.L. Detection of atypical porcine pestivirus in Brazil in the central nervous system of suckling piglets with congenital tremor. Transbound Emerg. Dis. 2018, 65, 375-380. [CrossRef]

10. Mósena, A.C.S.; Weber, M.N.; da Cruz, R.A.S.; Cibulski, S.P.; da Silva, M.S.; Puhl, D.E.; Hammerschmitt, M.E.; Takeuti, K.L.; Driemeier, D.; de Barcellos, D.E.S.N.; et al. Presence of atypical porcine pestivirus (APPV) in Brazilian pigs. Transbound Emerg. Dis. 2018, 65, 22-26. [CrossRef]

11. Postel, A.; Hansmann, F.; Baechlein, C.; Fischer, N.; Alawi, M.; Grundhoff, A.; Derking, S.; Tenhündfeld, J.; Pfankuche, V.M.; Herder, V.; et al. Presence of atypical porcine pestivirus (APPV) genomes in newborn piglets correlates with congenital tremor. Sci. Rep. 2016, 6, 27735. [CrossRef] [PubMed]

12. Beer, M.; Wernike, K.; Dräger, C.; Höper, D.; Pohlmann, A.; Bergermann, C.; Schröder, C.; Klinkhammer, S.; Blome, S.; Hoffmann, B. High Prevalence of Highly Variable Atypical Porcine Pestiviruses Found in Germany. Transbound Emerg. Dis. 2017, 64, e22-e26. [CrossRef] [PubMed]

13. de Groof, A.; Deijs, M.; Guelen, L.; van Grinsven, L.; van Os-Galdos, L.; Vogels, W.; Derks, C.; Cruijsen, T.; Geurts, V.; Vrijenhoek, M.; et al. Atypical Porcine Pestivirus: A Possible Cause of Congenital Tremor Type A-II in Newborn Piglets. Viruses 2016, 8, 271. [CrossRef] [PubMed]

14. Yuan, J.; Han, Z.; Li, J.; Huang, Y.; Yang, J.; Ding, H.; Zhang, J.; Zhu, M.; Zhang, Y.; Liao, J.; et al. Atypical Porcine Pestivirus as a Novel Type of Pestivirus in Pigs in China. Front. Microbiol. 2017, 8, 862. [CrossRef] [PubMed]

15. Postel, A.; Meyer, D.; Cagatay, G.N.; Feliziani, F.; De Mia, G.M.; Fischer, N.; Grundhoff, A.; Milićević, V.; Deng, M.C.; Chang, C.Y.; et al. High Abundance and Genetic Variability of Atypical Porcine Pestivirus in Pigs from Europe and Asia. Emerg. Infect. Dis. 2017, 23, 2104-2107. [CrossRef] [PubMed]

16. Muñoz-González, S.; Canturri, A.; Pérez-Simó, M.; Bohórquez, J.A.; Rosell, R.; Cabezón, O.; Segalés, J.; Domingo, M.; Ganges, L. First report of the novel atypical porcine pestivirus in Spain and a retrospective study. Transbound Emerg. Dis. 2017, 64, 1645-1649. [CrossRef] [PubMed]

17. Cagatay, G.N.; Antos, A.; Meyer, D.; Maistrelli, C.; Keuling, O.; Becher, P.; Postel, A. Frequent infection of wild boar with atypical porcine pestivirus (APPV). Transbound Emerg. Dis. 2018, 65, 1087-1093. [CrossRef]

18. Colom-Cadena, A.; Ganges, L.A.; Muñoz-González, S.; Castillo-Contreras, R.; Alejandro Bohórquez, J.; Rosell, R.; Segalés, J.; Marco, I.; Cabezon, O. Atypical porcine pestivirus in wild boar (Sus scrofa), Spain. Vet. Rec. 2018, 183, 569.

19. Schwarz, L.; Riedel, C.; Högler, S.; Sinn, L.J.; Voglmayr, T.; Wöchtl, B.; Dinhopl, N.; Rebel-Bauder, B.; Weissenböck, H.; Ladinig, A.; et al. Congenital infection with atypical porcine pestivirus (APPV) is associated with disease and viral persistence. Vet. Res. 2017, 48, 1. [CrossRef]

20. OIE Manual for Terrestrial Animals 2016 cap 2.1.4 par B1, B1.1, B1.2, B1.3: Brucellosis [Brucella abortus, B. melitensis, B. suis (Infection with B. abortus, B. melitensis, B. suis)]—Diagnostic Techniques_Identification of the Agent-Staining Methods-Collection of Samples and Culture-Identification and Typing. Available online: https://www.oie.int/fileadmin/Home/eng/Health_standards/tahm/3.01.04_BRUCELLOSIS. pdf (accessed on 15 October 2019).

21. Ehricht, R.; Slickers, P.; Goellner, S.; Hotzel, H.; Sachse, K. Optimized DNA microarray assay allows detection and genotyping of single PCR-amplifiable target copies. Mol. Cell Probes. 2006, 20, 60-66. [CrossRef]

22. Vicari, N.; Santoni, R.; Vigo, P.G.; Magnino, S. A PCR-RFLP assay targeting the 16 S ribosomal gene for the diagnosis of animal chlamydioses. In Proceedings of the 5th Meeting of the European Society for Chlamydia Research, Budapest, Hungary, 1-4 September 2004.

23. Van Kuppeveld, F.J.; van der Logt, J.T.; Angulo, A.F.; van Zoest, M.J.; Quint, W.G.; Niesters, H.G.; Galama, J.M.; Melchers, W.J. Genus- and species-specific identification of mycoplasmas by $16 \mathrm{~S}$ rRNA amplification. Appl. Environ. Microbiol. 1992, 58, 2606-2615, Erratum in: Appl. Environ. Microbiol. 1993, 59, 655. 
24. Olvera, A.; Sibila, M.; Calsamiglia, M.; Segalés, J.; Domingo, M. Comparison of porcine circovirus type 2 load in serum quantified by a real time PCR in postweaning multisystemic wasting syndrome and porcine dermatitis and nephropathy syndrome naturally affected pigs. J. Virol. Methods 2004, 117, 75-80. [CrossRef] [PubMed]

25. Kim, J.; Choi, C.; Han, D.U.; Chae, C. Simultaneous detection of porcine circovirus type 2 and porcine parvovirus in pigs with PMWS by multiplex PCR. Vet. Rec. 2001, 149, 304-305. [CrossRef] [PubMed]

26. Palinski, R.; Pineyro, P.; Shang, P.; Yuan, F.; Guo, R.; Fang, Y.; Byers, E.; Hause, B.M. A novel porcine circovirus distantly related to known circoviruses is associated with porcine dermatitis and nephropathy syndrome and reproductive failure. J. Virol. 2016, 91, 16. [CrossRef] [PubMed]

27. OIE Manual for Terrestrial Animals 2018 cap 3.4.7 par B1.2: Bovine Viral Diarrhea-Diagnostic Techniques-Detection of the Agent-Nucleic Acid Detection. Available online: https://www.oie.int/ fileadmin/Home/eng/Health_standards/tahm/3.04.07_BVD.pdf (accessed on 15 October 2019).

28. Brocchi, E.; Cordioli, P.; Berlinzani, A.; Gamba, D.; De Simone, F. Development of a panel of anti-pestivirus monoclonal antibodies useful for virus identification and antibody assessment. In Proceedings of the second Symposium on Ruminant Pestiviruses, Annecy, France, 1-3 October 1992; Edwards, S., Ed.; Fondation Marcel Mérieux: Lyon, France, 1993; pp. 215-218.

29. Grazioli, S.; Pezzoni, G.; Cordioli, P.; Brocchi, E. Validation of a competitive ELISA for serodiagnosis of PRRS based on recombinant n-protein and monoclonal antibody. In Proceedings of the 8th International Congress of Veterinary Virology, Budapest, Hungary, 24-27 August 2009; Benkõ, M., Harrach, B., Eds.; Hungarian Academy of Sciences: Budapest, Hungary, 2009; p. 199.

30. Heckert, R.A.; Brocchi, E.; Berlinzani, A.; Mackay, D.K. An international comparative analysis of a competitive ELISA for the detection of antibodies to swine vesicular disease virus. J. Vet. Diagn. Invest. 1998, 10, $295-297$. [CrossRef]

31. OIE Manual for Terrestrial Animals 2018 cap 3.8.8 par B2.2: Swine Vesicular Disease-Diagnostic Techniques-Serological Tests_Enzyme-Linked Immunosorbent Assay. Available online: https://www.oie. int/fileadmin/Home/eng/Health_standards/tahm/3.08.08_SVD.pdf (accessed on 15 October 2019).

32. Brocchi, E.; Carra, E.; Koenen, F.; De Simone, F. Sviluppo di metodi ELISA basati sull'impiego di anticorpi monoclonali per la dimostrazione del virus della encefalomiocardite (EMCV) e dei relativi anticorpi; La Selezione Veterinaria-Supplemento; IZSLER: Brescia, Italy, 2000; pp. 207-215.

33. OIE Manual for Terrestrial Animals 2018 cap 3.1.2 par B2.2: Aujeszky's Disease (Infection with Aujeszky's Disease Virus)—Diagnostic Techniques—Serological Tests—Enzyme-Linked Immunosorbent Assay (a Prescribed Test for International Trade). Available online: https://www.oie.int/fileadmin/Home/eng/ Health_standards/tahm/3.01.02_AUJESZKYS.pdf (accessed on 15 October 2019).

34. OIE Manual for Terrestrial Animals 2019 cap 3.8.3 par B2.3: Classical Swine Fever (Infection with Classical Swine Fever Virus)—Diagnostic Techniques_-Serological Tests_Enzyme-Linked Immunosorbent Assay (a Prescribed Test for International Trade). Available online: https://www.oie.int/fileadmin/Home/eng/ Health_standards/tahm/3.08.03_CSF.pdf (accessed on 15 October 2019).

35. Kendall, A.P.; Pereira, M.S.; Skehel, J.J. Concepts and Procedures for Laboratory-Based Influ-Enza Surveillance; World Health Organization: Geneva, Switzerland, 1982; (Copies available from the WHO Collaborating Centre for Surveillance, Epidemiology and Control of Influenza, CDC, Atlanta, GA).

36. OIE Manual for Terrestrial Animals 2018 cap 3.8.7 par B2.1: Influenza A Virus of Swine-Diagnostic Techniques-Serological Tests—Haemagglutination Inhibition Test. Available online: https://www.oie.int/ fileadmin/Home/eng/Health_standards/tahm/3.08.07_INF_A_SWINE.pdf (accessed on 15 October 2019).

37. GenBank ${ }^{\circledR}$ (NCBI). Available online: www.ncbi.nlm.nih.gov (accessed on 22 November 2019).

38. Tamura, K.; Stecher, G.; Peterson, D.; Filipski, A.; Kumar, S. MEGA6: Molecular evolutionary genetics analysis version 6.0. Mol. Biol. Evol. 2013, 30, 2725-2729. [CrossRef]

39. Nguyen, L.T.; Schmidt, H.A.; von Haeseler, A.; Minh, B.Q. IQ-TREE: A fast and effective stochastic algorithm for estimating maximum-likelihood phylogenies. Mol. Biol. Evol. 2015, 32, 268-274. [CrossRef]

40. Kalyaanamoorthy, S.; Minh, B.Q.; Wong, T.K.F.; von Haeseler, A.; Jermiin, L.S. ModelFinder: Fast model selection for accurate phylogenetic estimates. Nat. Methods 2017, 14, 587-589. [CrossRef]

(C) 2019 by the authors. Licensee MDPI, Basel, Switzerland. This article is an open access article distributed under the terms and conditions of the Creative Commons Attribution (CC BY) license (http://creativecommons.org/licenses/by/4.0/). 